\title{
Análisis de la evolución del modelo de proteína en los libros de texto de ESO y Bachillerato españoles
}

\author{
Beatriz Mazas Gil iD \\ Dpto. de Didácticas Especificas. Área de Didáctica de las Ciencias experimentales. Universidad de Zaragoza. \\ España.bmazas@unizar.es
}

Esther Cascarosa Salillas

Dpto. de Didácticas Especificas. Área de Didáctica de las Ciencias experimentales. Universidad de Zaragoza. España.ecascano@unizar.es

Ángel Luis Cortés Gracia iD
Dpto.de Didácticas Especificas. Área de Didáctica de las Ciencias experimentales. Universidad de Zaragoza.
España: acortes@unizar.es

[Recibido: 16 enero 2020. Revisado: 9 junio 2020. Aceptado: 6 julio 2020]

Resumen: En este trabajo se realiza un análisis de contenido de libros de texto de Biología y Geología, desde $1^{\circ}$ ESO a $2^{\circ}$ Bachillerato, en relación a la evolución que sufre el modelo de proteína. Se han empleado 20 libros de editoriales de amplia distribución en las aulas de todo el país. Durante el análisis se han elaborado una serie de modelos parciales por cursos que recogen los elementos que se tienen en cuenta en cada nivel, atendiendo a los contenidos que aparecen en los libros de texto que, establecidos en diferentes categorías, se van ampliando curso a curso hasta llegar a un modelo complejo de proteína en $2^{\circ}$ Bachillerato. Se ha observado que, dependiendo del libro empleado en el aula, los estudiantes podrían tener un modelo más o menos completo en los cursos más bajos, pues no todos los libros se ciñen exclusivamente a los contenidos curriculares correspondientes, sino que van introduciendo algunos aspectos que se verán en cursos posteriores y que permiten al alumno contextualizar mejor el modelo que van creando, favoreciendo el pensamiento sistémico.

Palabras clave: Educación Secundaria; libros de texto; modelos; proteína.

\section{Analysis of the evolution of protein's model within the Spanish Secondary textbooks}

Abstract: In this work we have analysed the contents of textbooks of Biology and Geology from $7^{\text {th }}$ Grade to $12^{\text {th }}$ Grade, regarding the evolution about the model of protein. We have used 20 textbooks of wide distribution in Spain highschools. During analysing we have made some partial models that gather the elements taken into account in each level. Considering the textbook contents in different categories, the model grows to get a complex model of protein in the $12^{\text {th }}$ Grade. We have observed that students could have a more or less complete model of protein if the textbook is one or another in the lower levels, because not every textbook is just showing the curricular contents, but they also introduce some aspects they will learn in next levels. That allows the students to have a context for the model they are making, contributing to systemic thinking.

Keywords: Secondary Education; textbooks; models; protein.

Para citar este artículo: Mazas B., Cascarosa E. y Cortés A. (2020) Análisis de la evolución del modelo de proteína en los libros de texto de ESO y Bachillerato españoles. Revista Eureka sobre Enseñanza y Divulgación de las Ciencias 17(3), 3103. doi: 10.25267/Rev_Eureka_ensen_divulg_cienc.2020v17.i3.3103

\section{Introducción}

El término proteína es común en el vocabulario coloquial, ahora bien, ¿qué es exactamente una proteína?, ¿para qué sirven las proteínas?, ¿qué alimentos contienen proteínas?, ¿hay unas proteínas mejores que otras?, ¿cómo se sintetizan las proteínas? Así, podríamos hacer una lista interminable de preguntas para conocer qué significa este concepto para los alumnos de Educación Secundaria Obligatoria (en adelante ESO) y/o Bachillerato. El currículo español de

\author{
Revista Eureka sobre Enseñanza y Divulgación de las Ciencias \\ Universidad de Cádir. APAC-Eureka. ISSN: 1697-011X \\ bttp:/ / dx.doi.org/10.25267/Rev_Eureka_ensen_divulg_cienc.2020.v17.i3.3103 \\ bttp:/ / reuredc.uca.es
}


secundaria (Real Decreto 1105/2014) presenta contenidos relacionados con las proteínas desde $1^{\circ}$ ESO hasta $2^{\circ}$ Bachillerato, por lo que una vez terminadas estas etapas, los estudiantes han tenido oportunidad para conocer muchas cosas sobre ellas. La situación es similar en otros países y los resultados de la investigación educativa sugieren que muchos estudiantes tienen problemas para comprender el concepto de proteína y para conectarlo con otros aspectos de la genética y la biología molecular desde un punto de vista sistémico (Lewis y Kattmann 2004, Thörne y Gericke 2014). Pero lo que es más importante, la formación recibida en relación a este concepto debería dar a los estudiantes las herramientas necesarias para tener criterios suficientes que les permitan interpretar hechos y fenómenos de su día a día. Por ejemplo, en relación a la alimentación, el concepto de proteína se utiliza al hablar de las dietas, pero ¿conocen los alumnos por qué es necesario consumir proteínas?, ¿cuáles son los efectos sobre el cuerpo? En la enseñanza reglada se trabaja el concepto de proteína, pero ¿se construye un modelo que permita desarrollar la competencia en ámbitos concretos como el de la alimentación?, es decir, ¿el modelo construido en relación a la alimentación es directamente aplicable en su vida cotidiana? España, Cabello y Blanco (2014) analizaron las destrezas que deberían desarrollar los alumnos a lo largo de la educación obligatoria para alcanzar competencias en alimentación, y destacan la importancia de saber conceptualizar los alimentos, por lo que, para poder decidir sobre una alimentación adecuada, hay que conocer qué son (entre otros componentes/nutrientes) las proteínas.

Desde el punto de vista educativo, a los alumnos se les debe facilitar la alfabetización científica, lo que, entre otras cosas, les ayudará a ser críticos con la información que reciben en su día a día. La adquisición de contenido científico facilita el desarrollo del pensamiento crítico, por tanto, en este contexto, la construcción y comprensión del modelo de proteína ayudará a los alumnos en ese sentido.

El modelo de proteína de los alumnos se construye, entre otras cosas, a partir de los contenidos que aparecen en los libros de texto, ya que siguen siendo una herramienta fundamental en las aulas (Martínez y Rodríguez 2010, Mazas Gil y Fernández Manzanal 2016). Por ello, es importante conocer el modelo de proteína que se muestra en los mismos. Según algunos autores, los libros de texto concretan los significados de los contenidos incluidos en el currículo, tratando de facilitar la enseñanza y aprendizaje de los mismos a través de adaptaciones basadas en criterios pedagógicos (Gimeno 2005, Gómez 2016, entre otros). No obstante, es tal la importancia en nuestro sistema educativo que "este recurso ha sido algo más que un material de aprendizaje: se ha confundido con el currículo oficial..." (Rodríguez et al. 2018, p. 3102-2). Por ello, en esta investigación destacamos la importancia de conocer también lo que marca la ley en relación al tema de estudio. Como indican algunos autores, los libros de texto no siempre se adaptan adecuadamente al currículo en todas las dimensiones del mismo (Calvo y Martín 2005). También el currículo limita en parte la construcción del modelo del alumno ya que únicamente se articula en torno a una lista de contenidos conceptuales (Martínez y García 2003). Occelli y Valeiras (2013) estudiaron en detalle publicaciones nacionales e internacionales (en una ventana temporal de diez años) donde se analizaba la relación entre los contenidos del currículo y los de los libros de texto, concluyendo en posibles mejoras de los contenidos trabajados desde estos últimos, por ejemplo tratando de vincular unos contenidos con otros, o estableciendo categorías entre lo trabajado en un curso y en el siguiente que faciliten al alumno retomar lo trabajado en cursos anteriores.

Además, también es interesante conocer si el modelo de proteína y su evolución depende del libro de texto que se use. Antes de realizar el análisis de contenido, se ha revisado el currículo aragonés de ESO y Bachillerato, para determinar cuándo, dónde y cómo aparecen los contenidos relacionados con las proteínas. A continuación, se ha analizado en profundidad una muestra de 20 libros de texto de Biología y Geología en todos los cursos de ESO y 
Bachillerato, poniendo el foco en el modelo de proteína. De esta manera, podemos formular la siguiente cuestión que guiará nuestra investigación: ¿qué modelos de proteína aparecen y cómo evoluciona el concepto de proteína en los diferentes libros de texto? Tomando como base este análisis del contenido sobre proteínas en los libros de texto, se propone el modelo de proteína que se podría construir en cada uno de los cursos analizados.

\section{Modelos y libros de texto}

Un modelo es una representación de un objeto o un fenómeno con un objetivo específico (Gilbert, Boulter y Elmer 2000, Oliva 2019). Según Giere (2004, 2010), las personas producen o usan un modelo para representar una parte del mundo con algún propósito, es decir, el modelo no representa nada por sí mismo, sino que la representación tiene lugar cuando alguien usa el modelo. La estrategia de enseñanza y aprendizaje basada en modelos se fundamenta en la concepción de éstos como núcleo central del conocimiento científico y la modelización como principal proceso para construir y utilizar ese conocimiento (Gilbert y Justi 2016). Así, en palabras de Oliva (2019, p. 6) "la educación científica se interpreta en clave de aprendizaje de modelos, orientación que parece la más frecuente en los estudios realizados en el marco de la modelización". Un proceso de construcción del modelo adecuado debería permitir que los estudiantes apliquen de forma correcta el modelo en una situación diferente a la de aprendizaje, aunque sea muy distinta (Uskola, Burgoa y Maguregi 2018) y, además, la práctica de modelización en el aula permite a los docentes acceder a las ideas del alumnado y conocer cómo evolucionan a través de la comunicación de sus modelos mentales (Mendonça y Justi 2014).

Los términos modelo y modelización tienen distintas acepciones y usos. Autores como Gilbert y Justi (2016) diferencian entre la enseñanza basada en modelos (model-based teaching), o uso de los modelos existentes por los estudiantes, y la enseñanza basada en la modelización (modelling-based teaching), que supone la creación y el uso de modelos por parte de los estudiantes. Oliva (2019) hace una revisión profunda de la literatura existente, identificando hasta cinco acepciones distintas para el término modelización dependiendo de su papel en el proceso de enseñanza y aprendizaje de las ciencias: 1) como progresión de modelos, 2) como práctica científica, 3) como competencia, 4) en su dimensión instrumental y 5) como enfoque didáctico. En este trabajo tiene especial relevancia el planteamiento que hace este autor de la modelización como progresión de modelos, entendida como "oportunidad de encontrar sentido global a los conocimientos que aprenden los estudiantes, y de avanzar paulatinamente hacia una comprensión más ajustada de la realidad" (Oliva 2019, p. 16).

La elaboración de un modelo mental es una actividad que puede desarrollarse individualmente o en grupo y que puede ser representada mediante acciones como el habla, la escritura u otras formas simbólicas (Bahamonde y Gómez-Galindo 2016). A esta representación es a lo que se denomina modelo expresado (Gilbert y Boulter 1995). El proceso de construcción de modelos mentales se genera mediante los razonamientos y experiencias que se lleven a cabo y es señalado como clave en el aprendizaje de las ciencias en la escuela (Justi 2006). El análisis de la adecuación del modelo construido por el alumno puede hacerse a través de una exposición descriptiva o interpretativa del concepto trabajado (Bahamonde y Gómez-Galindo 2016). Atendiendo a las ventajas que supone para el alumnado que el profesor diseñe el proceso de enseñanza alrededor de la construcción de modelos, cada vez se está tratando de implantar esta propuesta de enseñanza en más centros educativos y así lo demuestran algunas investigaciones (Maguregi, Uskola y Burgoa 2017, Hester et al. 2018), aunque sigue existiendo una enorme brecha entre la investigación y la práctica educativa en las aulas de Primaria y Secundaria (Oliva 2019). Sin embargo, existen muy pocos trabajos hasta la fecha donde se haya analizado el modelo mental que los alumnos tienen sobre el concepto de proteína (Barak y 
Hussein-Farraj 2013). Algunos autores llevaron a cabo una investigación educativa en torno a la comprensión que los alumnos tienen del concepto de proteína, concluyendo que, nos solo tienen un modelo de proteína erróneo y/o incompleto, sino que tienen dificultades para conectarlo con otros aspectos de la genética y la biología molecular desde un punto de vista sistémico (Lewis y Kattmann 2004, Thörne y Gericke 2014). Otros autores, como Íñiguez y Puigcerver (2013), recogen las dificultades que tienen los alumnos para relacionar las proteínas por ejemplo con el material hereditario; Ageitos, Puig y Calvo-Peña (2017) describen la dificultad para construir un modelo de gen que facilite la comprensión de ciertas enfermedades; y Ohly (1998) señala dificultades de los estudiantes a la hora de entender la genética molecular por contener gran cantidad de conceptos abstractos y microscópicos.

En este contexto, los libros de texto juegan un papel fundamental en el proceso de enseñanza y aprendizaje de los modelos científicos (Harrison 2001), ya que representan la progresión de los modelos de las diferentes disciplinas tomando como referencia el currículo escolar (Torres 1989, Clement 2000). De esta forma, en los últimos años se han abordado numerosos estudios centrados en el contenido científico y didáctico de los textos escolares (Occelli y Valeiras 2013), siendo especialmente interesantes para nuestro estudio aquellos que analizan los modelos incluidos en esos libros (Jiménez, Prieto y Perales 1997, Harrison 2001, Pozzer y Roth 2003, Fernández, González y Moreno 2005, Gericke y Hagberg 2010). Algunos de los problemas que detectan estos trabajos son, entre otros, la falta de claridad e incoherencias en los conceptos implicados (Martínez-Gracia, Gil-Quílez y Osada 2006), la falta de actividades para la aplicación de esos modelos en los libros de texto (Puig y Jiménez-Aleixandre 2015) y, en concreto, en relación con el tema que nos ocupa, aparece un enfoque fundamentalmente conceptual, centrado en las estructuras generales de la síntesis de proteínas (Wahlberg y Gericke 2018).

Por todo lo anterior, y porque tanto la investigación en didáctica como los nuevos marcos curriculares de ciencias destacan la importancia de la construcción y uso de modelos (Couso 2014) así como la participación del alumnado en las prácticas científicas (Schwarz et al. 2009), este trabajo trata de analizar la evolución del modelo de proteína en los libros de texto. Teniendo en cuenta que, en su mayor parte, el modelo de proteína que construyen los alumnos se basa en los modelos que aparecen en los libros de texto, los objetivos fundamentales de este trabajo se centran en: 1) determinar los contenidos sobre proteínas presentes en 5 colecciones completas de ESO y Bachillerato, 2) construir modelos de proteína para cada uno de los cursos de ESO y Bachillerato atendiendo a los contenidos de los diferentes libros de texto, y 3) analizar la evolución de dichos modelos en las diferentes editoriales.

\section{Metodología}

Como paso previo al análisis de los libros de texto se ha realizado una revisión del currículo LOMCE de ESO y Bachillerato (Real Decreto 1105/2014). Hasta $2^{\circ}$ de Bachillerato no aparece el término de forma explícita en el currículo como contenido, pero el resto de cursos hemos revisado aquellos contenidos que tuvieran una relación implícita o explícita con las proteínas. Por ejemplo, entendemos que podrían mostrarse proteínas en los libros de texto si se habla de biomoléculas orgánicas, o en general de los seres vivos, o de nutrientes orgánicos/nutrición, o de genética en los procesos de transcripción-traducción... Para conocer cómo se ha trasladado el modelo de proteína desde distintas perspectivas a los libros de texto de ESO y Bachillerato, se ha procedido a realizar un análisis de contenido (Bardin 1986, López 2002) a partir de una muestra de 20 libros de algunas de las principales editoriales de amplia distribución nacional (Santillana, SM, Vicens Vives y Anaya). Se han analizado cuatro colecciones completas de cinco libros cada una correspondientes a $1^{\circ}, 3^{\circ}$ y $4^{\circ}$ ESO y $1^{\circ}$ 
y $2^{\circ}$ Bachillerato, que corresponden a los cursos donde aparecen en el currículo contenidos relacionados con las proteínas en Biología.

Se han revisado estos contenidos en el libro de texto para ver qué incluyen sobre proteínas: en primer lugar, si aparece el término, y en su caso, si se construyen modelos más complejos que pueden introducir aspectos como por ejemplo la composición, las funciones, las estructuras, etc.

A partir de los resultados de esta revisión, se ha establecido un sistema de categorías que ha servido como referencia para la elaboración de las plantillas de análisis de contenido de los libros de texto. Como indican algunos autores, el análisis de contenido "sólo puede entenderse de una forma completa si lo situamos en el contexto de un diseño de investigación" (López 2002, p. 173) y, de esta forma, la definición de las unidades de análisis y categorías es uno de sus principales problemas. Por ello, no existen plantillas de análisis generalizables, sino que en cada análisis es necesario inventar o adaptar la técnica más adecuada para los intereses de la investigación (Perales y Jiménez 2002, Mampel y Cortés 2012).

En $1^{\circ}$ ESO las categorías son diferentes del resto de cursos, porque la relación con las proteínas es esporádica en algunos casos y, como hemos indicado, no se nombran como tal en el currículo. Para este curso se han definido las siguientes categorías de análisis: 1) aparece el término de proteína, 2) forman parte de los seres vivos, 3) nutrientes orgánicos, 4) funciones, 5) modelo de proteína y 6) ejemplos. Por tanto, en la primera de ellas, lo que se ha buscado es la presencia del término proteína de forma explícita dentro de los contenidos. Cuando lo hace, está normalmente asociado a la materia viva con las biomoléculas, y observamos si incluyen las proteínas como parte de los seres vivos. La tercera categoría implica que se consideren como un nutriente orgánico, es decir, que se obtenga de los alimentos. La cuarta está relacionada con que se señale alguna de las funciones de las proteínas (distintas de la función energética de la categoría anterior). En la quinta categoría se contempla si existe alguna imagen o modelo de algún tipo de proteína y, finalmente, se ha incluido una categoría de ejemplos en la que se explicite específicamente una proteína en un contexto concreto.

En el resto de los cursos hemos contemplado otras categorías estables, aunque se han desarrollado diferentes subcategorías adaptadas en cada curso, en función de los contenidos específicos declarados en el currículo. Estas categorías son: 1) unidades fundamentales, 2) estructura y funciones, 3) ¿cómo se sintetizan?, 4) modelo de proteína y 5) ejemplos. A continuación, se describe cada una de ellas:

- Con unidades fundamentales nos referimos a los aminoácidos (en adelante, áá), que son la base de las proteínas. Así en $3^{\circ}$ ESO se plantea como subcategoría que el libro recoja que las proteínas son la unión de áá, hasta 20 distintos, que aparezca el concepto de aminoácido esencial, y que los áá se obtienen a partir de dietas saludables. En $4^{\circ}$ ESO aparecen subcategorías como la organización celular, que implica que se refieren a las proteínas en algún momento cuando se habla de la organización de la célula y los orgánulos; o la secuencia de aminoácidos que da lugar a hasta 20 áá distintos. En Bachillerato se considera si se nombra el carbono alfa, y si especifica que los áá son distintos gracias al radical R. También se incluye como subcategoría que se encuentre la estructura atómica de los aminoácidos y sus propiedades, así como la unión entre ellos, es decir, el enlace peptídico.

- En la categoría estructura y funciones, en $3^{\circ}$ ESO, además de la estructura, las funciones se clasifican en dos subcategorías: la energía como nutriente, entendida como la función energética de las proteínas; u otras funciones, como la enzimática o la plástica. En $4^{\circ}$ ESO se agrupan estructura y funciones ya que la estructura se relaciona con la función. En Bachillerato se tienen en cuenta como subcategorías los enlaces, la actividad biológica, el 
proceso de desnaturalización, la descripción individual de cada una de los niveles estructurales de las proteínas y, por último, si aparecen ejemplos de cada función específica.

- En la categoría relacionada con ¿cómo se sintetizan? se proponen varias subcategorías. En $3^{\circ}$ ESO se tiene en cuenta la idea de fábrica de proteínas y que la digestión de las mismas comienza en el estómago. En $4^{\circ}$ ESO se atiende a que las proteínas son diferentes en función del orden, del número de aminoácidos y del tipo que sean, y otra subcategoría engloba la síntesis proteica en los ribosomas. En Bachillerato se subdivide con respecto a la traducción, a que se haga la diferenciación entre péptido, polipéptido y proteína, o a que se clasifiquen en holo y heteroproteínas.

- En la categoría modelo de proteína, al igual que en $1^{\circ} \mathrm{ESO}$, se considera si se muestra alguna imagen o representación que responda a un modelo de algún tipo de proteína.

- Finalmente, la categoría de ejemplos se ha establecido en los cursos de $3^{\circ}$ y $4^{\circ}$ ESO. En $3^{\circ}$, haciendo alusión a ejemplos de proteínas o de alimentos proteicos; en $4^{\circ}$, atendiendo a ejemplos de proteína o bien de enfermedades genéticas. En Bachillerato no se ha considerado oportuno porque constantemente afloran ejemplos que corresponden a las otras categorías.

Atendiendo a los contenidos de los libros analizados, se representa en los resultados, a modo de esquema gráfico, el modelo de proteína de cada uno de los cursos, donde se puede apreciar la progresiva complejidad en cuanto a los contenidos relacionados con las proteínas. En cada figura (correspondiente a cada curso), en torno al concepto central de proteína (situado en el centro de color naranja) se representan una serie de globos que representan los subcomponentes del modelo. Los colores asignados en cada globo de contenido se mantienen a lo largo de los cursos, entendiéndose que el color representa contenidos próximos o con cierta relación: función, estructura, biomoléculas, nutrición, células, clasificación y ejemplos (en este último, junto con subcategorías en globos más pequeños) los cuales se especifican en la leyenda que se presenta en la Figura 1.

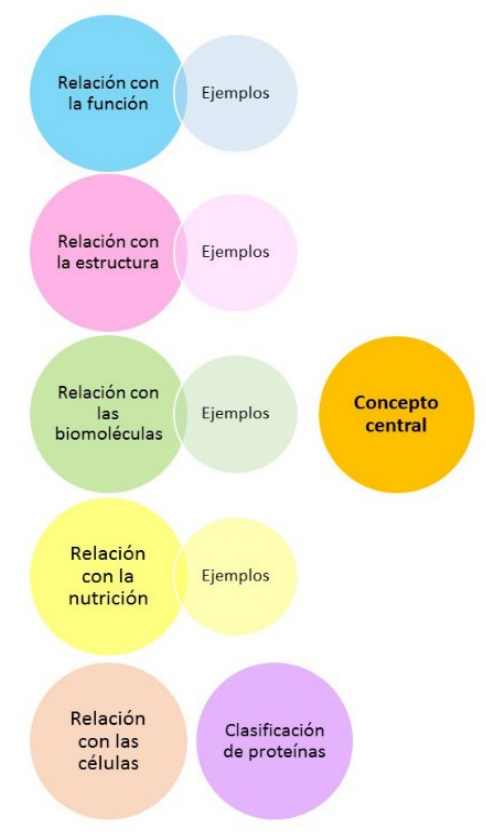

Figura 1. Leyenda de los elementos y colores de los modelos representados en los diferentes cursos 


\section{Análisis de los libros de texto}

\section{Primero de ESO}

En $1^{\circ}$ ESO las proteínas se engloban dentro del bloque 3 sobre biodiversidad en el planeta, donde se indica que los seres vivos están constituidos por células y cuáles son sus características, así como las funciones comunes, entre las que se encuentra la nutrición (autótrofa y heterótrofa).

Dependiendo del libro seleccionado aparecen diferentes aproximaciones a las proteínas. En la tabla 1 se puede apreciar que en todos los libros analizados se encuentra el término proteína, que es un nutriente orgánico que forma parte de los seres vivos. En el libro A se indica que las proteínas pueden ser de muchos tipos distintos y que intervienen en multitud de procesos, destacando las funciones estructurales. El libro B se refiere a las proteínas como sustancias ricas en carbono que abundan en el músculo de los animales. En el libro C, se definen las biomoléculas y presentan una tabla en la que se indica donde abundan, su función y las características, así como una foto en la que se muestran alimentos que tienen mayor cantidad de proteínas. En este caso se aprecia un acercamiento mayor a las proteínas también en otros aspectos. Por ejemplo, aparecen imágenes realistas de proteínas en la membrana plasmática, modelos estructurales de proteínas como la hemoglobina, ejemplos de los alimentos que las contienen o las necesidades diarias recomendadas de proteína. El libro D se refiere a las proteínas como biomoléculas orgánicas, sin profundizar más. También hay que señalar que tres de los cuatro libros (A, B y C), al introducir el tema de las células procariotas, indican que los ribosomas son los orgánulos que se encargan de fabricar las proteínas.

Teniendo en cuenta el contenido respecto a las proteínas en los diferentes libros analizados, se propone un modelo de proteína de $1^{\circ} \mathrm{ESO}$ atendiendo los conceptos que se consideran en los diferentes libros de texto (Figura 2).

Tabla 1. Análisis de los libros de $1^{\circ}$ ESO

\begin{tabular}{|l|c|c|c|c|}
\hline Categorías / Libros & Libro A & Libro B & Libro C & Libro D \\
\hline Aparece el término de proteína & $\mathrm{x}$ & $\mathrm{x}$ & $\mathrm{x}$ & $\mathrm{x}$ \\
\hline Forman parte de los seres vivos & $\mathrm{x}$ & $\mathrm{x}$ & $\mathrm{x}$ & $\mathrm{x}$ \\
\hline Nutrientes orgánicos & $\mathrm{x}$ & $\mathrm{x}$ & $\mathrm{x}$ & $\mathrm{x}$ \\
\hline Funciones & $\mathrm{x}$ & $\mathrm{x}$ & $\mathrm{x}$ & \\
\hline Modelo de proteína & & & $\mathrm{x}$ & \\
\hline Ejemplos & & & $\mathrm{x}$ & \\
\hline
\end{tabular}

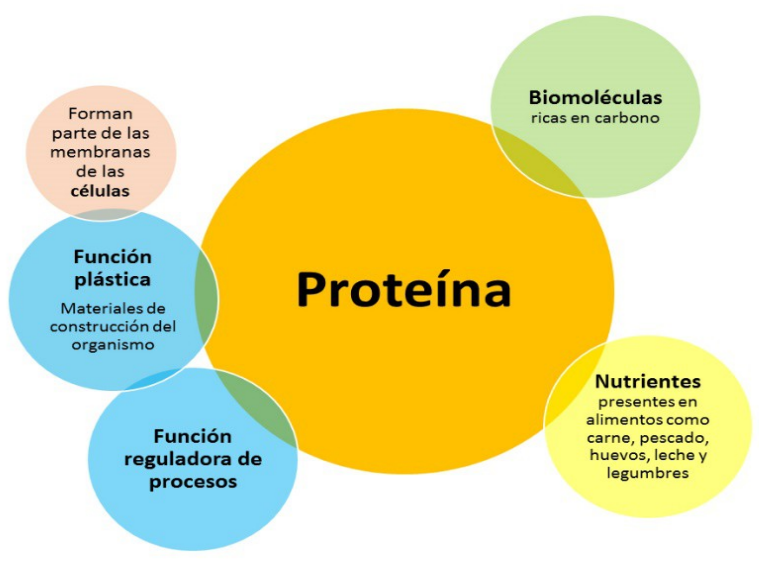

Figura 2. Componentes del modelo de proteína de $1^{\circ}$ ESO 


\section{Tercero de ESO}

En $3^{\circ}$ ESO las proteínas se engloban dentro del bloque de biodiversidad, como moléculas que forman parte de los seres vivos, que nos sirven como nutrientes y, por tanto, están presentes en los alimentos. También se señalan como contenido las dietas saludables. Así pues, entendemos que se plantea una relación más próxima con el concepto de nutrición.

En los libros $\mathrm{E}$ y $\mathrm{F}$ se atiende al porcentaje de proteínas necesario para tener una dieta equilibrada (15\%), y se indica que los alimentos ricos en proteínas (carne, pescado, legumbres) tienen una función plástica. Los libros $\mathrm{G}$ y $\mathrm{H}$ además añaden que, en una dieta equilibrada, el contenido en proteínas de origen animal no debe sobrepasar más del 40\% del total. Los libros F, G y H detallan aspectos que no se corresponden con los contenidos que indica el currículo para este curso. Por ejemplo, indican que el material genético tiene las instrucciones para fabricar proteínas (libro G), aunque este tema se inicia al hablar de los ribosomas como orgánulos y señalan que éstos son la fábrica de las proteínas (libro $\mathrm{H}$ ). En los libros F y $\mathrm{G}$ también se indica cómo se fabrica una proteína digestiva en el tema de La célula. En estos libros adelantan contenidos sobre este tema, que será clave en el siguiente curso.

Tabla 2. Análisis de los libros de $3^{\circ} \mathrm{ESO}$

\begin{tabular}{|c|c|c|c|c|c|}
\hline Categorías & Subcategorías & Libro E & Libro F & Libro G & Libro $\mathrm{H}$ \\
\hline \multirow{4}{*}{ Unidades fundamentales } & Unión de aminoácidos & $\mathrm{x}$ & $\mathrm{x}$ & $\mathrm{x}$ & $\mathrm{x}$ \\
\hline & $\begin{array}{ll}20 & \text { aminoácidos } \\
\text { distintos } & \\
\end{array}$ & & $\mathrm{x}$ & $\mathrm{x}$ & $\mathrm{x}$ \\
\hline & Aminoácidos esenciales & & & $\mathrm{x}$ & $\mathrm{x}$ \\
\hline & Dieta saludable & & $\mathrm{x}$ & $\mathrm{x}$ & $\mathrm{x}$ \\
\hline \multirow{3}{*}{ Estructura y funciones } & Estructura & & & & \\
\hline & Energía como nutriente & & $\mathrm{x}$ & & $\mathrm{x}$ \\
\hline & $\begin{array}{l}\text { Otras funciones: } \\
\text { enzimática, plástica }\end{array}$ & $\mathrm{x}$ & $\mathrm{x}$ & $\mathrm{x}$ & $\mathrm{x}$ \\
\hline \multirow[b]{2}{*}{ ¿Cómo se sintetizan? } & Fábrica de proteínas & $\mathrm{x}$ & $\mathrm{x}$ & $\mathrm{x}$ & $\mathrm{x}$ \\
\hline & $\begin{array}{l}\text { Digestión comienza en } \\
\text { el estómago }\end{array}$ & $\mathrm{x}$ & $\mathrm{x}$ & $\mathrm{x}$ & \\
\hline Modelo de proteína & $\begin{array}{l}\text { Representación de un } \\
\text { modelo de proteína }\end{array}$ & $\mathrm{x}$ & $\mathrm{x}$ & $\mathrm{x}$ & $\mathrm{x}$ \\
\hline \multirow[b]{2}{*}{ Ejemplos } & Ejemplos de proteína & $\mathrm{x}$ & $\mathrm{x}$ & & $\mathrm{x}$ \\
\hline & $\begin{array}{l}\text { Ejemplos de alimentos } \\
\text { proteicos }\end{array}$ & $\mathrm{x}$ & $\mathrm{x}$ & $\mathrm{x}$ & $\mathrm{x}$ \\
\hline
\end{tabular}

En este curso, en todos los libros analizados se muestran imágenes que representan modelos parciales de proteína, que, si bien no abarcan todo el contenido, facilitan la comprensión del texto al que acompañan. En la figura 3 se plantea el modelo de proteína que corresponde a $3^{\circ}$ ESO considerando los contenidos clave que aparecen en los libros de texto, podríamos decir que son biomoléculas formadas por áá, que hay veinte distintos y que algunos de ellos son esenciales. A partir de la información genética, que se lee en los ribosomas, se sintetizan las distintas proteínas. Algunas de sus funciones son: la energética, donde se señalan los alimentos con mayor proporción de proteínas como la carne y el pescado, las legumbres, los huevos o la leche y derivados, así como la energía que aporta $1 \mathrm{~g}$ de proteínas; la función enzimática, indicando proteínas como la pepsina, y que la digestión de las proteínas comienza en el estómago; y, finalmente la función plástica, donde se indica que las proteínas forman parte de las membranas de las células. 


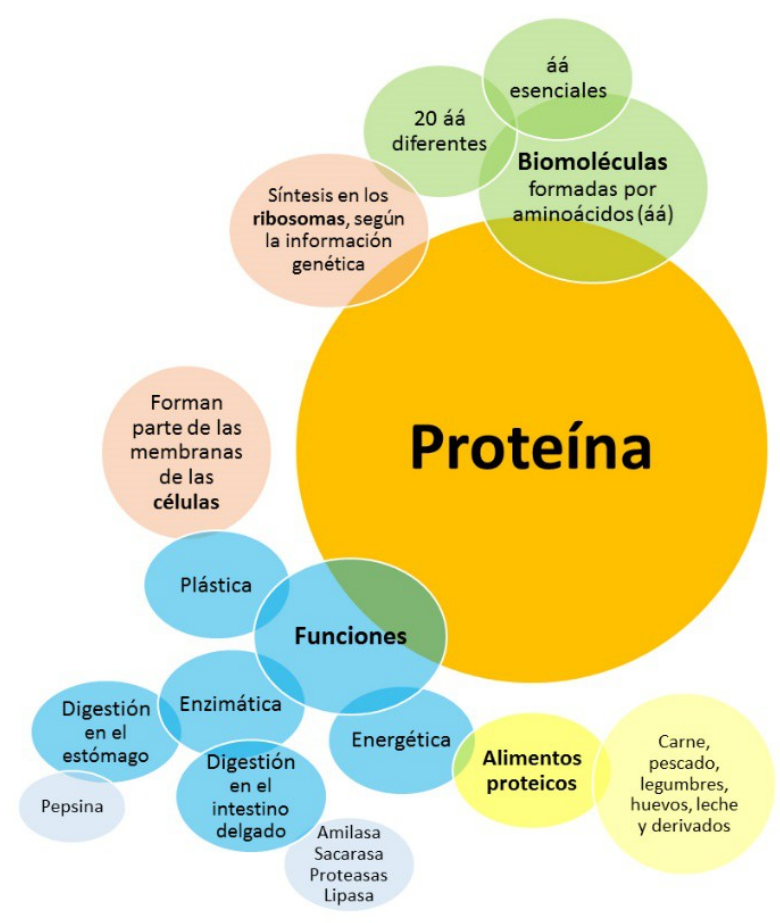

Figura 3. Componentes del modelo de proteína de $3^{\circ}$ ESO

\section{Cuarto de ESO}

En $4^{\circ}$ ESO la orientación del tema deriva hacia la genética, donde las proteínas resultan de la transcripción y traducción del ADN.

En la tabla 3 se presenta el análisis de las categorías en $4^{\circ}$ ESO. El tema al que están asociadas las proteínas es la genética, por lo que el modo de abordarlas en todos los libros está ligado a los procesos de transcripción y traducción del $\mathrm{ADN}$, es decir, a la síntesis de proteínas. Se hace explícito en todos los textos el hecho de que los genes contienen la información para construir una proteína concreta. En algunos casos se ponen ejemplos, como en el libro J, donde se ve una aplicación directa en un error genético de una enfermedad concreta (fenilcetonuria), y en otros -como I, K y L- se hace alusión a la fábrica de proteínas, que son los ribosomas, en el tema de la célula. En los libros K y L se habla, además, de la estructura de las proteínas y de las funciones, como que intervienen en el metabolismo o que forman parte de estructuras celulares. Sobre todo, en el libro $\mathrm{K}$ se ponen ejemplos de proteínas como hemoglobina, anticuerpos, actina y miosina, insulina, enzimas... al referirse a la expresión de la información del ADN.

Tabla 3. Análisis de los libros de $4^{\circ} \mathrm{ESO}$

\begin{tabular}{|c|c|c|c|c|c|}
\hline Categorías & Subcategorías & Libro I & Libro J & $\begin{array}{c}\text { Libro } \\
\text { K }\end{array}$ & $\begin{array}{c}\text { Libro } \\
\text { L }\end{array}$ \\
\hline \multirow{3}{*}{$\begin{array}{l}\text { Unidades } \\
\text { fundamentales }\end{array}$} & Organización celular & $\mathrm{x}$ & & $\mathrm{x}$ & $\mathrm{x}$ \\
\hline & Secuencia de aminoácidos & $\mathrm{x}$ & & $\mathrm{x}$ & \\
\hline & 20 aminoácidos distintos & $\underline{x}$ & & $\mathrm{x}$ & $\mathrm{x}$ \\
\hline $\begin{array}{l}\text { Estructura y } \\
\text { funciones }\end{array}$ & Estructura y funciones & & & $\mathrm{x}$ & $\mathrm{x}$ \\
\hline \multirow[b]{2}{*}{ ¿Cómo se sintetizan? } & Proteínas diferentes (según orden, $\mathrm{n}^{\circ}$, tipo) & $\mathrm{x}$ & & $\mathrm{x}$ & $\mathrm{x}$ \\
\hline & $\begin{array}{llll}\text { Síntesis proteica } & \text { (traducción en los } \\
\text { ribosomas) }\end{array}$ & $\mathrm{x}$ & $\mathrm{x}$ & $\mathrm{x}$ & $\mathrm{x}$ \\
\hline \multirow{2}{*}{ Ejemplos } & Ejemplos de proteínas & & $\mathrm{x}$ & $\mathrm{x}$ & \\
\hline & Enfermedades genéticas & $\mathrm{x}$ & $\mathrm{x}$ & $\mathrm{x}$ & $\mathrm{x}$ \\
\hline
\end{tabular}


Para representar el modelo de $4^{\circ}$ ESO (Figura 4) se han tenido en cuenta los aspectos que más se repiten en los libros de texto. En primer lugar, la formación de las proteínas, que es el contenido fundamental de dicho curso. Por otro lado, lo que se recuerda de cursos anteriores, como la descripción del concepto de proteína y algunas funciones. Y, finalmente, algunos ejemplos contemplados de forma secundaria y aquellos conceptos que no aparecen explícitamente en el currículo como, por ejemplo, la estructura.

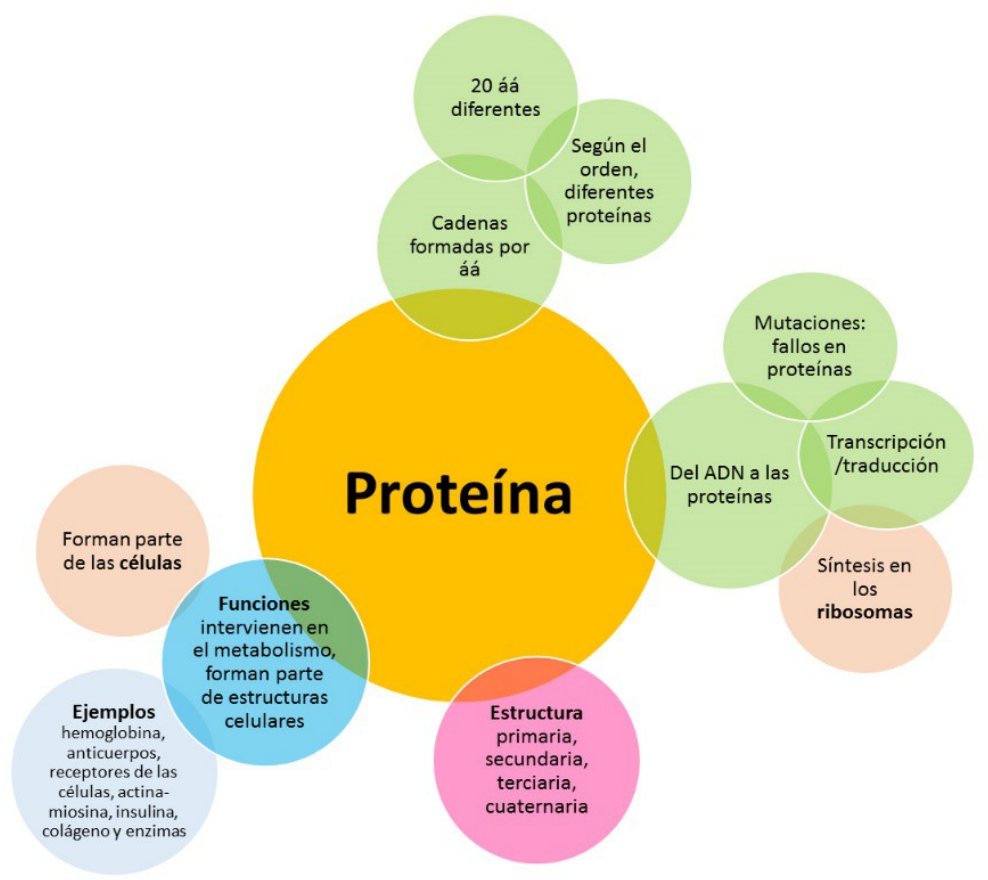

Figura 4. Componentes del modelo de proteína de $4^{\circ}$ ESO

\section{Bachillerato}

En $1^{\circ}$ Bachillerato se habla de las proteínas en el bloque de los seres vivos. En él, se describe cada una de las diferentes biomoléculas que constituyen la materia viva y la función que realizan. A nivel de $2^{\circ}$ Bachillerato se detalla la base molecular de la vida y se dedican temas completos a las macromoléculas. Por tanto, se profundiza en los monómeros, los enlaces que los unen, las funciones que desempeñan, su composición química, su función y su formación a partir de la transcripción y traducción del $\mathrm{ADN}$, ampliando los contenidos que se impartieron en todos los cursos anteriores.

Se presenta una tabla única para los dos cursos de Bachillerato, ya que muchos de los conceptos se repiten o se amplían de $1^{\circ}$ a $2^{\circ}$ Bachillerato (Tabla 4). No obstante, se profundiza mucho más en $2^{\circ}$, por lo que a continuación se comenta cada uno por separado. 
Tabla 4. Análisis de los libros de $1^{\circ}$ y $2^{\circ}$ de Bachillerato

\begin{tabular}{|c|c|c|c|c|c|c|c|c|c|}
\hline \multirow{2}{*}{ Categorías } & \multirow{2}{*}{ Subcategorías } & \multicolumn{4}{|c|}{$1^{\circ}$ Bachillerato } & \multicolumn{4}{|c|}{$2^{\circ}$ Bachillerato } \\
\hline & & $\mathbf{M}$ & $\mathbf{N}$ & $\mathbf{O}$ & $\mathbf{P}$ & $\mathbf{Q}$ & $\mathbf{R}$ & $\mathrm{S}$ & $\mathbf{T}$ \\
\hline \multirow{7}{*}{$\begin{array}{c}\text { Unidades } \\
\text { fundamentales }\end{array}$} & Carbono alfa & $\mathrm{x}^{*}$ & $\mathrm{x}$ & $x^{*}$ & $\mathrm{x}$ & $\mathrm{x}$ & $\mathrm{x}$ & $\mathrm{x}$ & $\mathrm{x}$ \\
\hline & Radical R: 20 áá distintos & $\mathrm{x}$ & $\mathrm{x}$ & $\mathrm{x}$ & $\mathrm{x}$ & $\mathrm{x}$ & $\mathrm{x}$ & $\mathrm{x}$ & $\bar{x}$ \\
\hline & áá esenciales & & & $\mathrm{x}$ & & $\mathrm{x}$ & $\mathrm{x}$ & $\mathrm{x}$ & $\mathrm{x}$ \\
\hline & Alimentos con áá-esenciales & $\mathrm{x}$ & $\bar{x}$ & & & $\mathrm{x}$ & $\mathrm{x}$ & $\bar{x}$ & $\bar{x}$ \\
\hline & Estructura atómica áá & $\mathrm{x}$ & $\mathrm{x}$ & $\mathrm{x}$ & $\mathrm{x}$ & $\mathrm{x}$ & $\mathrm{x}$ & $\mathrm{x}$ & $\mathrm{x}$ \\
\hline & Propiedades de los áá & & & & & $\mathrm{x}$ & $\mathrm{x}$ & $\mathrm{x}$ & $\mathrm{x}$ \\
\hline & Enlace peptídico & $\mathrm{x}$ & $\mathrm{x}$ & $\mathrm{x}$ & $\mathrm{x}$ & $\mathrm{x}$ & $\mathrm{x}$ & $\mathrm{x}$ & $\mathrm{x}$ \\
\hline \multirow{5}{*}{$\begin{array}{l}\text { Estructura y } \\
\text { funciones }\end{array}$} & Enlaces & $\mathrm{x}$ & $\mathrm{x}$ & $\mathrm{x}$ & & $\mathrm{x}$ & $\mathrm{x}$ & $\mathrm{x}$ & $\mathrm{x}$ \\
\hline & Actividad biológica & $\mathrm{x}$ & & & & $\mathrm{x}$ & $\mathrm{x}$ & $\mathrm{x}$ & $\mathrm{x}$ \\
\hline & Desnaturalización & $\mathrm{x}$ & $\mathrm{x}$ & $\mathrm{x}$ & & $\mathrm{x}$ & $\mathrm{x}$ & $\mathrm{x}$ & $\mathrm{x}$ \\
\hline & $\begin{array}{l}\text { Descripción individual de las } \\
\text { estructuras }\end{array}$ & & & $\mathrm{x}$ & & $\mathrm{x}$ & $\mathrm{x}$ & $\mathrm{x}$ & $\mathrm{x}$ \\
\hline & Ejemplos de función & $\mathrm{x}$ & $\mathrm{x}$ & $\mathrm{x}$ & $\mathrm{x}$ & $\mathrm{x}$ & $\mathrm{x}$ & $\mathrm{x}$ & $\mathrm{x}$ \\
\hline \multirow{3}{*}{$\begin{array}{l}\text { ¿Cómo se } \\
\text { sintetizan? }\end{array}$} & Traducción & & $\mathrm{x}$ & & $\mathrm{x}$ & & $\mathrm{x}$ & $\mathrm{x}$ & \\
\hline & $\begin{array}{l}\text { Péptido/ } \\
\text { polipéptido/proteína }\end{array}$ & $\mathrm{x}$ & $\mathrm{x}$ & $\mathrm{x}$ & $\mathrm{x}$ & $\mathrm{x}$ & $\mathrm{x}$ & $\mathrm{x}$ & $\mathrm{x}$ \\
\hline & $\begin{array}{l}\text { Holoproteínas / } \\
\text { Heteroproteínas }\end{array}$ & & & & & $\mathrm{x}$ & $\mathrm{x}$ & $\mathrm{x}$ & $\mathrm{x}$ \\
\hline $\begin{array}{l}\text { Modelo de } \\
\text { proteína }\end{array}$ & & & $\mathrm{x}$ & $\mathrm{x}$ & & $\mathrm{x}$ & $\mathrm{x}$ & $\mathrm{x}$ & $\mathrm{x}$ \\
\hline
\end{tabular}

Dos de los libros analizados de $1^{\circ}$ Bachillerato ( $\mathrm{M}$ y $\mathrm{P}$ ) presentan contenidos similares sobre proteínas. Coinciden en la descripción de la estructura atómica, la variabilidad en cuanto a la cadena R, la estructura de las proteínas y las funciones más importantes. También incluyen ejemplos en cada una de ellas, como la función de defensa (anticuerpos, fibrinógeno y trombina para la coagulación) o la estructural (el colágeno de los tendones, la queratina de las uñas o las glucoproteínas de las membranas celulares). En el libro M no aparece un modelo gráfico de proteína como tal, y en el libro P lo representan a partir de las enzimas en la explicación de su mecanismo de actuación. Sin embargo, sí que se muestran modelos de proteína como la hemoglobina en los libros $\mathrm{N}$ y O. En el libro $\mathrm{N}$ y en el $\mathrm{P}$ reaparece la síntesis de las proteínas (vista en el curso anterior) al hablar de los ácidos nucleicos, por lo que se describen la transcripción y la traducción más profundamente en este tema.

En cuanto al modelo de proteína propuesto en este curso (Figura 5), se atiende especialmente a la composición más detallada de los aminoácidos y a las funciones de las proteínas. En este modelo se introduce la idea de que la pérdida de la estructura conlleva la pérdida de la función (desnaturalización). Se relaciona también el hecho de que los aminoácidos son diferentes gracias a la variabilidad que les proporciona la cadena $\mathrm{R}$, que se determina atendiendo a los codones específicos que se traducen en los ribosomas, según viene determinado en los genes. 


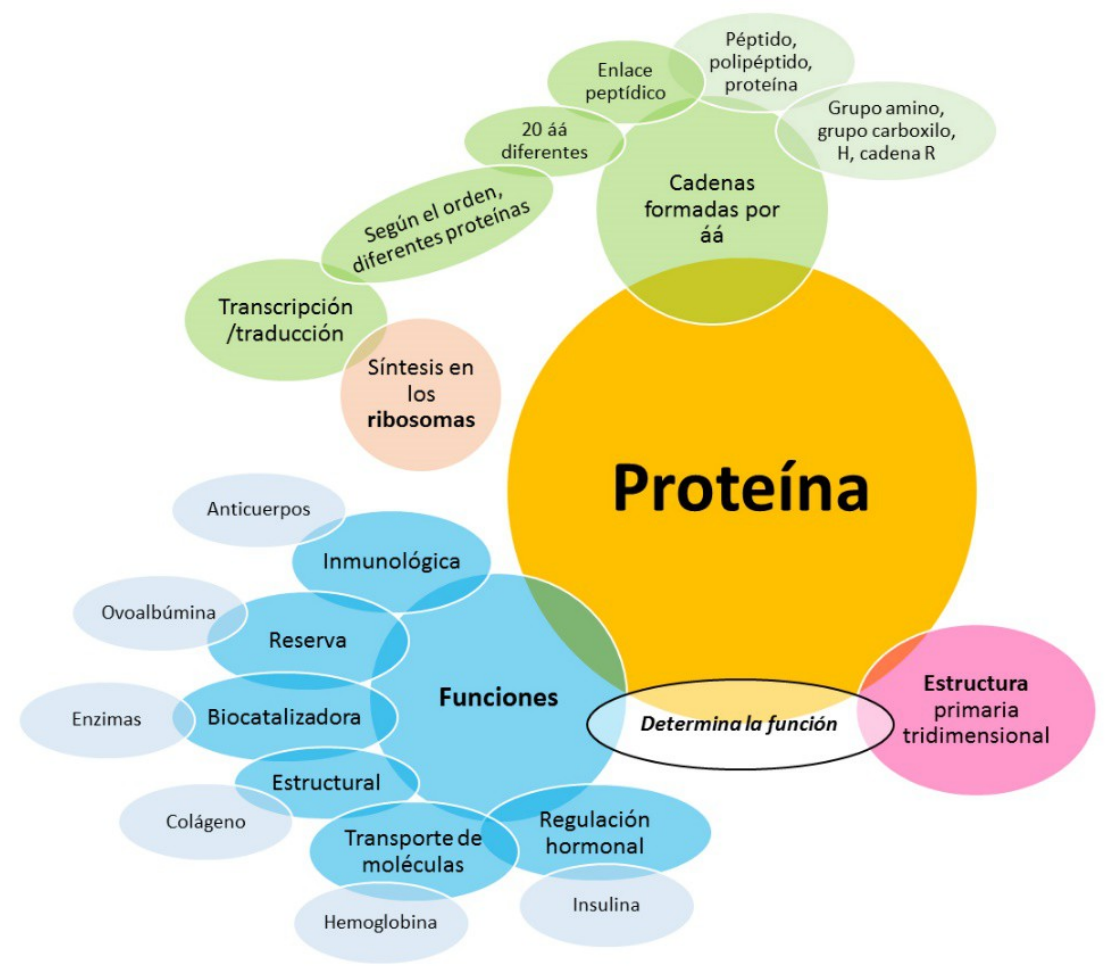

Figura 5. Componentes del modelo de proteína de $1^{\circ}$ Bachillerato

En $2^{\circ}$ Bachillerato se amplían los contenidos que se estudiaron en $1^{\circ}$, como la descripción de los aminoácidos, el carbono alfa, la profundización sobre los aminoácidos esenciales, el enlace peptídico, las propiedades de los aminoácidos y, sobre todo, la descripción detallada de la estructura primaria, secundaria, terciaria y cuaternaria de las proteínas. También se incide en el desarrollo de las funciones y la clasificación en cuanto a que sean holo o heteroproteínas. Asimismo, se mantienen al margen otros aspectos como el origen de las proteínas, por lo que no se relaciona explícitamente con los procesos de transcripción y traducción (visto en $4^{\circ}$ ESO), salvo brevemente en el libro S. Tampoco se incide en los alimentos que contenían mayor proporción de proteínas (visto en $3^{\circ} \mathrm{ESO}$ ), aunque solamente se refleja una pequeña reseña sobre el huevo en los libros $\mathrm{R}$ y $\mathrm{S}$. El libro $\mathrm{S}$ introduce el valor biológico de las proteínas con ejemplos de distintos alimentos con mayor o menor valor biológico. En el libro T se inicia la unidad exponiendo el contexto histórico de los aminoácidos y de diversas proteínas. También indica que los enlaces peptídicos se rompen por hidrólisis, algo imprescindible para comprender cómo se obtiene energía de las proteínas y cómo pueden formarse y deshacerse dichos enlaces. Hay que señalar que en este libro $T$ aparece una abundante descripción de la actividad enzimática. En este curso, en todos los libros hay multitud de representaciones que tienen que ver con los diferentes procesos en los que intervienen las proteínas, su funcionalidad (ejemplo enzima sustrato), así como la representación atómica del enlace peptídico.

En la Figura 6 se propone el modelo de proteína que se considera para $2^{\circ}$ Bachillerato que, como se puede comprobar, es el más complejo de los presentados. En ese modelo se insertan multitud de elementos que se abordan en profundidad, desde la composición química y las propiedades de los aminoácidos, los enlaces que se producen entre ellos, el listado de los 20 aminoácidos y cuáles son esenciales (en la Figura 6 aparecen subrayados), hasta el desarrollo de las estructuras de las proteínas y las funciones que desempeñan, con ejemplos de cada una de ellas. También se contempla la función de nutrición, que se rescata de $3^{\circ}$ ESO donde se 
trataba más profundamente, considerando qué alimentos son ricos en proteínas. Del mismo modo se introducen los conceptos de holo y heteroproteínas que hasta ahora no habían aparecido.

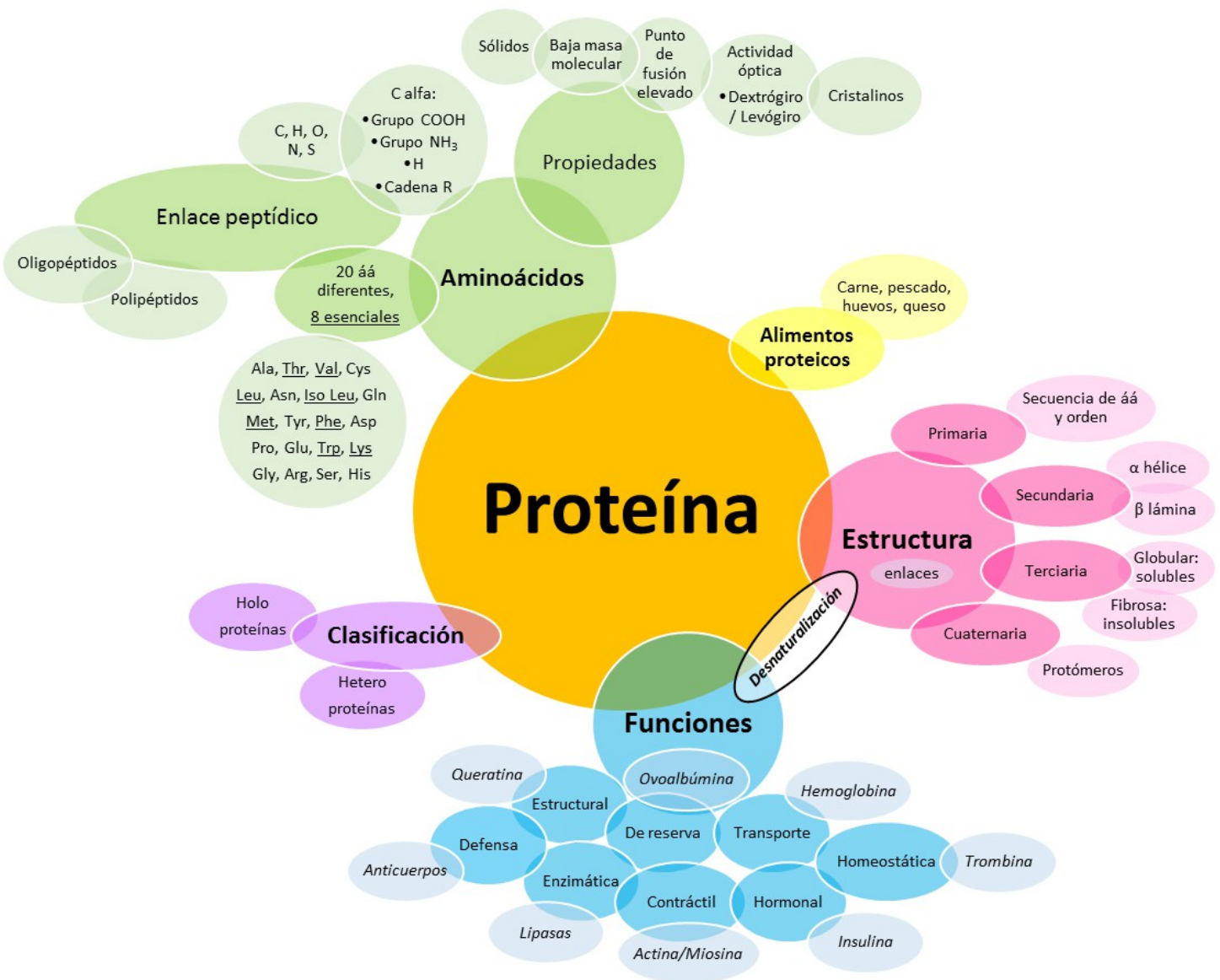

Figura 6. Componentes del modelo de proteína de $2^{\circ}$ Bachillerato

Atendiendo a la evolución que hacen las editoriales en los modelos de proteína que se plantean, siguiendo los contenidos relacionados en cada uno de los cursos, podemos señalar que los libros C, G, K O y S, pertenecientes a la Editorial Vicens Vives, son los que presentan una evolución más completa del modelo, contextualizando progresivamente con conceptos en los que se va a profundizar en los cursos superiores.

\section{Discusión}

Atendiendo a los resultados presentados, podemos señalar que no todos los libros de texto analizados incorporan los mismos conceptos, ni lo hacen siguiendo la misma secuencia, a pesar de que diversos autores indican que desarrollar la enseñanza a través de una secuencia didáctica bien establecida facilita la construcción de modelos (Oliva 2019). Hay libros de texto, como es el caso del libro $\mathrm{C}$ de $1^{\circ} \mathrm{ESO}$, que engloban más aspectos que los propiamente curriculares (Real Decreto 1105/2014), ahora bien, ¿'es necesario? Algunos estudios previos (Martínez- Gracia, Gil-Quílez y Osada 2006) señalan que los libros de texto deberían ajustarse a la descripción de los procesos como la transcripción, la síntesis proteica y la regulación génica, simplificando aspectos innecesarios para favorecer una mejor comprensión de los conceptos básicos, apropiados al nivel de los estudiantes. 
En este estudio sobre las proteínas, a diferencia de lo que comentaban los autores anteriores, consideramos necesario que aparezcan contenidos anejos para entender el contexto general en el que aparecen las proteínas, y sobre todo aquellos elementos o procesos no visibles y que se vinculan con mayores dificultades de comprensión, por ser más abstractos (Ohly 1998), como por ejemplo la función de los genes en la síntesis proteica (Walberg y Gericke 2018). Con esto no queremos decir que haya que incluir en los libros de texto de los primeros cursos contenidos complejos que no son apropiados para su nivel educativo, sino que, como se ha visto en algunos de los libros analizados, se pueden ir contextualizando y englobando algunos conceptos que tienen que ver con las proteínas y que se verán en profundidad en cursos superiores. De esta forma, los alumnos pueden progresar en la construcción de un modelo parcial, pero cada vez más elaborado, de proteína desde el comienzo de su etapa secundaria.

La construcción del modelo se irá formando de este modo, aglutinando nuevos conceptos, pero con una base sólida que permita incorporar nuevos contenidos sobre los ya existentes. Es por ello que en este trabajo se han presentado los modelos parciales que se recogen en cada uno de los cursos como una progresión de contenidos de aprendizaje interrelacionados entre sí, según aparecen en los libros de texto analizados.

\section{Conclusiones e implicaciones educativas}

Atendiendo a los objetivos planteados, se ha revisado en qué cursos y partes del currículo aparecen contenidos relacionados con las proteínas. El análisis de contenido ha permitido determinar que los diferentes libros de texto analizados tratan el concepto de proteína a lo largo de toda la etapa secundaria, sufriendo una evolución progresiva en la que se van incorporando conceptos cada vez más complejos desde $1^{\circ}$ ESO hasta $2^{\circ}$ Bachillerato, siendo que el término proteína no aparece de forma explícita en el currículo hasta $2^{\circ}$ de Bachillerato. Pero no todos los libros de texto muestran la misma evolución en el modelo de proteína.

En cuanto al segundo objetivo, se han construido los modelos de proteína por cursos. A modo de resumen podemos señalar que en $1^{\circ} \mathrm{ESO}$ el concepto de proteína queda relegado a la idea de nutriente orgánico, en $3^{\circ}$ el modelo se articula alrededor de la nutrición, en $4^{\circ}$ se centra en la formación de las proteínas desde los genes y en Bachillerato incluye todos los aspectos trabajados, los engloba en un modelo complejo y los amplía hasta niveles moleculares, de enlaces, de estructura y de funciones, con mucho detalle. Los modelos que presentamos pueden servir como orientación para trabajar las proteínas como una progresión de contenidos desde $1^{\circ}$ ESO hasta $2^{\circ}$ Bachillerato a través de la cual los estudiantes puedan ser capaces de entender aquellas situaciones que se les plantean en su entorno y reflexionar sobre su propia comprensión acerca de los conceptos clave que se relacionan con las proteínas desde una perspectiva sistémica.

Con respecto al último de los objetivos, la evolución del modelo a lo largo de cada una de las cuatro editoriales analizadas, se ha observado que, a lo largo de la ESO, los modelos que plantean los libros $\mathrm{C}$ (en $\left.1^{\circ} \mathrm{ESO}\right), \mathrm{F}$ (en $3^{\circ} \mathrm{ESO}$ ) y K (en $4^{\circ} \mathrm{ESO}$ ) son los más completos. En dichos textos se introducen la mayor parte de los conceptos relacionados con las proteínas (del acuerdo al nivel educativo). Y lo mismo ocurre con los libros $\mathrm{O}$ y $\mathrm{S}$ en $1^{\circ}$ y $2^{\circ}$ Bachillerato, respectivamente. Por tanto, la progresión utilizada en la editorial Vicens Vives podría ayudar a la construcción de un modelo más complejo sobre proteína por parte de los alumnos.

Otra consideración relevante que queremos resaltar es que en los libros de texto no se muestran de manera explícita las relaciones entre los aminoácidos que se digieren, la célula y las nuevas proteínas. Esto quiere decir que no queda claro que los aminoácidos incorporados a través de la dieta (y que son los que se utilizan en la traducción) son los que forman parte de las proteínas que se sintetizan. Esto podría solventarse evitando hablar, por un lado, de la 
traducción y, por otro lado, de la digestión, que es lo que se ha observado en este estudio. Por lo tanto, a pesar de que hay libros de texto que facilitan la construcción del modelo, todavía aparecen conceptos no relacionados que pueden dificultar que el alumno comprenda el concepto de proteína desde una perspectiva más amplia.

Por lo tanto, del presente estudio sobre el modelo de proteína se puede concluir que, en el caso de un enfoque didáctico basado en el uso de libros de texto, la cantidad de contenidos y la secuencia planteada para trabajarlos dependerá del libro concreto que se esté utilizando en un curso determinado, ya que los contenidos no son equivalentes. Por otro lado, se ha encontrado que las referencias al concepto de proteína se hacen, en la mayor parte de los casos, desde una perspectiva antropocéntrica, es decir, se habla mucho de las proteínas del cuerpo humano o las que necesitamos los seres humanos, pero menos de las vegetales y apenas de las proteínas presentes en otros seres vivos, a pesar de que algunos de los libros señalan que el porcentaje de proteínas de origen animal no debe suponer más de un $40 \%$ de las proteínas totales de la dieta. Sin embargo, no se abunda en esta idea. Todo ello podría estar dificultando la construcción de un modelo complejo y completo de proteína en el alumnado.

Entendemos que, tal y como señalan Íñiguez y Puigcerver (2013), la explicación de estos modelos debe utilizar referentes cercanos al alumnado, para que los estudiantes se impliquen en la construcción de su modelo mental (y/o expresado), relacionándolo con experiencias que son frecuentes en su día a día. Por ejemplo, si la comida que consumen es rica en proteínas, si todos los alimentos con proteínas tienen las mismas o si las hay de diferentes calidades, ¿para qué sirven esas proteínas adicionales de la dieta que la publicidad o algunos referentes recomiendan consumir? Incluso podemos plantear pequeñas situaciones que permitan la comprensión del modelo completo y la relación de diferentes procesos relacionados con las proteínas en diferentes cursos como, por ejemplo: ¿para qué incorporamos proteínas en la dieta si ya las produce nuestro cuerpo a partir de los genes?

\section{Agradecimientos}

Al proyecto EDU2016-76743-P (Ministerio de Economía y Competitividad) y al Grupo de Referencia BEAGLE de Investigación en Didáctica de Ciencias Naturales (IUCA-Universidad de Zaragoza) financiado por el Gobierno de Aragón y cofinanciado con Feder 2014-2020 "Construyendo Europa desde Aragón". Al editor y los revisores por sus aportaciones que han ayudado a mejorar este trabajo.

\section{Referencias}

Bahamonde N., Gómez Galindo A.A. (2016) Caracterización de modelos de digestión humana a partir de sus representaciones y análisis de su evolución en un grupo de docentes y auxiliares académicos. Enseñanza de las Ciencias 34(1), 129-147. http://dx.doi.org/10.5565/rev/ensciencias.1748

Barak M., Hussein-Farraj R. (2013) Integrating model-based learning and animations for enhancing students' understanding of proteins structure and function. Research in Science Education 43(2), 619-636. https:/ / doi.org/10.1007/s11165-012-9280-7

Calvo Pascual M.A., Martín Sánchez M. (2005) Análisis de la adaptación de los libros de texto de ESO al currículo oficial, en el campo de la Química. Enseñanza de las Ciencias 23(1), $17-32$.

Clement J. (2000) Model based learning as a key research area for science education. International Journal of Science Education 22(9), 1041-1053. https://doi.org/10.1080/095006900416901 
Couso D. (2014) De la moda de "aprender indagando" a la indagación para modelizar: una reflexión crítica. En: M.A. Héras, A. Lorca, B. Vázquez, A. Wamba, R. Jiménez, Investigación y transferencia para una educación en ciencias: Un reto emocionante (pp. 1-28). Huelva: Servicio de Publicaciones Universidad de Huelva.

España Ramos E., Cabello Garrido A., Blanco López A. (2014) La competencia en alimentación. Un marco de referencia para la educación obligatoria. Enseñanza de las Ciencias 32(3), 611-629. http://dx.doi.org/10.5565/rev/ensciencias.1080

Fernández González J., González González B.M., Moreno Jiménez T. (2005) La modelización con analogías en los textos de ciencias de secundaria. Revista Eureka sobre Enseñanza y Divulgación de las Ciencias 2(3), 430-439. http://dx.doi.org/10.25267/Rev_Eureka_ensen_divulg_cienc.2005.v2.i3.10

Gericke N.M., Hagberg M. (2010) Conceptual Incoherence as a Result of the use of Multiple Historical Models in School Textbooks. Research in Science Education 40(4), 605-623. http://dx.doi.org/10.1007/s11191-012-9499-8

Giere R.N. (2004) How models are used to represent reality. Philosophy of Science 71, 742-752. http://dx.doi.org/10.1086/425063

Giere R.N. (2010) An agent-based conception of models and scientific representation. Synthese 172(2), 269-281. http://dx.doi.org/10.1007/S11229-009-9506-Z

Gilbert J.K., Boulter C.J. (1995) Stretching models too far. Comunicación presentada en Annual Meeting of the American Educational Research Association. San Francisco.

Gilbert J., Boulter C., Elmer R. (2000) Positioning Models in Science Education and in Design and Technology Education. En J. K. Gilbert y C. J. Boulter (Eds), Developing Models in Science Education (pp. 3-17). Dordrecht: Kluvet. https://doi.org/10.1007/978-94-0100876-1_1

Gilbert J.K., Justi R. (2016) Modelling-based Teaching in Science Education. Basel: Springer. http://doi.org/10.1007/978-3-319-29039-3

Gimeno Sacristán J. (2005) El currículum: ¿Los contenidos de la enseñanza o un análisis de la práctica? En: J. Gimeno Sacristán y A. I. Pérez Gómez (Eds.), Comprender y transformar la enseñanza (pp. 137-170). Madrid: Morata.

Gómez Mendoza M.A. (2016) El libro de texto escolar: espacios, lectura, hábitos digitales y recepción. Profesorado. Revista de currículum y formación de profesorado, 20(1), 33-47.

Harrison A.G. (2001) How do Teachers and Textbook Writers Model Scientific Ideas for Students? Research in Science Education 31, 401-435. https://doi.org/10.1023/A:1013120312331

Hester S.D., Nadler M., Katcher J., Elfring L.K., Dystra E., Rezende L.F., Bolger M.S. (2018) Authentic inquiry through modeling in biology (AIM-Bio): an introductory laboratory curriculum that increases undergraduates' scientific agency and skills. CBE-Life Sciences Education 17, 1-23. https://doi.org/10.1187/cbe.18-06-0090

Íñiguez Porras F.J., Puigcerver Oliván M. (2013) Una propuesta didáctica para la enseñanza de la genética en la Educación Secundaria. Revista Eureka sobre Enseñanza y Divulgación de las Ciencias 10(3), 307-327. http://dx.doi.org/10.25267/Rev_Eureka_ensen_divulg_cienc.2013.v10.i3.02

Jiménez J.D., Prieto R., Perales F.J. (1997) Análisis de los modelos y los grafismos utilizados en los libros de texto. Alambique 11, 75-85. 
Justi R. (2006) La enseñanza de ciencias basada en la elaboración de modelos. Enseñanza de las Ciencias 24(2), 173-184.

Lewis J., Kattmann U. (2004) Traits, genes, particles and information: re-visiting students' understandings of genetics. Research report. International Journal of Science Education 26(2), 195-206. https://doi.org/10.1080/0950069032000072782

López Noguero F. (2002) El análisis de contenido como método de investigación. XXI, Revista de Educación 4, 167-179.

Maguregi González G., Uskola Ibarluzea A., Burgoa Etxaburu B. (2017) Modelización, argumentación y transferencia de conocimiento sobre el sistema inmunológico a partir de una controversia sobre vacunación en futuros docentes. Enseñanza de las Ciencias 35(2), 29-50. http://dx.doi.org/10.5565/rev/ensciencias.2237

Mampel, L y Cortés, A. (2012). El concepto “dinosaurio" en los libros de texto. Enseñanza de las Ciencias de la Tierra, 20(3), 239-248.

Martínez Bonafé J., Rodríguez Rodríguez J. (2010) El curriculum y el libro de texto escolar. Una dialéctica siempre abierta. En J. Gimeno Sacristán (comp.), Saberes e incertidumbres sobre el curriculum (pp. 246-268). Madrid: Morata.

Martínez-Gracia M.V., Gil-Quílez M.J., Osada J. (2006) Analysis of molecular genetics content in Spanish secondary school textbooks. Journal of Biological Education 40(2), 53-60. http://dx.doi.org/10.1080/00219266.2006.9656014

Martínez Losada C., García Barros S. (2003) Las actividades de primaria y ESO incluidas en libros escolares. ¿Qué objetivo persiguen? ¿Qué procedimientos enseñan? Enseñanza de las Ciencias 21(2), 243-264.

Mazas Gil B., Fernández Manzanal R. (2016) El concepto de bienestar animal en el currículo de Secundaria Obligatoria y en los libros de texto de ciencias. Revista Eureka sobre Enseñanza y Divulgación de las Ciencias 13(2), 301-314. http://dx.doi.org/10498/18290

Mendonça P.C.C., Justi R. (2014) An instrument for analyzing arguments produced in modeling based chemistry lessons. Journal of Research in Science Teaching 51(2), 192-218. https://doi.org/10.1002/tea.21133

Occelli M., Valeiras N. (2013) Los libros de texto de ciencias como objeto de investigación: una revisión bibliográfica. Enseñanza de las Ciencias 31(2), 133-152. https://doi.org/10.5565/rev/ec/v31n2.761

Ohly K.P. (1998) Concepts and misconceptions in molecular genetics - a qualitative approach. En H. Bayrhuber y F. Brinkman, What-Why-How? Research in Didaktik of Biology. Proceedings of the First Conference of European Researchers in Didactic of Biology (ERIDOB) (pp. 184-195), Kiel, Germany: IPN.

Oliva J.M. (2019) Distintas acepciones para la idea de modelización en la enseñanza de las ciencias. Enseñanza de las Ciencias 37(2), 5-24. https://doi.org/10.5565/rev/ensciencias.2648

Perales F.J., Jiménez J. (2002) Las ilustraciones en la enseñanza-aprendizaje de las ciencias. Análisis de libros de texto. Enseñanza de las Ciencias 20(3), 369-386.

Pozzer L.L., Roth W.M. (2003) Prevalence, function and structure of photographs in high school biology textbooks. Journal of Research in Science Teaching 40(10), 1089-1114. https://doi.org/10.1002/tea.10122 
Puig B., Jiménez Aleixandre M.P. (2015) El modelo de expresión de los genes y el determinismo en los libros de texto de ciencias. Revista Eureka sobre Enseñanza y Divulgación le las Ciencias 12(1), 55-65. http://dx.doi.org/10.25267/Rev_Eureka_ensen_divulg_cienc.2015.v12.11.05

Real Decreto 1105/2014, LOMCE, https://www.boe.es/diario_boe/txt.php?id=BOE-A2015-37

Rodríguez Moreno J., Pro Bueno A., Molina Jaén M.D. (2018). Opinión de los docentes sobre el tratamiento de las competencias en los libros de texto de Ciencias de la Naturaleza en Educación Primaria. Revista Eureka sobre Enseñanza y Divulgación de las Ciencias, 18(3), 3102. https://doi.org/10.25267/Rev_Eureka_ensen_divulg_cienc.2018.v15.i3.3102

Schwarz C.V., Reiser B.J., Davis E.A., Kenyon L., Achér A., Fortus D., Shwartz Y., Hug B., Krajcik J. (2009) Developing a learning progression for scientific modeling: Making scientific modeling accessible and meaningful for learners. Journal of Research in Science Teaching 46(6), 632- 654. https:// doi.org/10.1002/tea.20311

Thörne K., Gericke N. (2014) Teaching Genetics in Secondary Classrooms: a Linguistic Analysis of Teachers' Talk About Proteins. Research in Science Education 44, 81-108. https://doi.org/10.1007/s11165-013-9375-9

Torres J. (1989) Libro de texto y control del currículum. Cuadernos de Pedagogía 168, 50-55.

Uskola A., Burgoa B., Maguregi G. (2018) Influencia de la ayuda del profesorado en la construcción del modelo de sistema inmunológico y su aplicación en las tomas de decisión. Revista Eureka sobre Enseñanza y Divulgación de las Ciencias 15(3), 3604. https://doi.org/10.25267/Rev_Eureka_ensen_divulg_cienc.2018.v15.i3.3604

Wahlberg S.J., Gericke N.M. (2018) Conceptual demography in upper secondary chemistry and biology textbooks' descriptions of protein synthesis: a matter of context? CBE-Life Sciences Education 17, 1-14. https://doi.org/10.1187/cbe.17-12-0274 


\section{Anexo 1}

Relación de códigos utilizados en el texto con su correspondiente editorial por cursos

\begin{tabular}{|c|c|c|c|c|}
\hline CURSO & $\begin{array}{l}\text { LETRA EN } \\
\text { EL TEXTO }\end{array}$ & EDITORIAL & AÑO & ISBN \\
\hline \multirow{4}{*}{$1^{\circ} \mathrm{ESO}$} & $\bar{A}$ & Santillana & 2015 & 978-84-680-1451-7 \\
\hline & B & $\mathrm{SM}$ & 2015 & $978-84-675-7609-2$ \\
\hline & $\mathrm{C}$ & Vicens Vives & 2015 & $978-84-682-3037-5$ \\
\hline & $\mathrm{D}$ & Anaya & 2015 & 978-84-678-5076-5 \\
\hline \multirow{4}{*}{$3^{\circ} \mathrm{ESO}$} & $\mathrm{E}$ & Santillana & 2015 & $978-84-294-7754-2$ \\
\hline & $\bar{F}$ & $\mathrm{SM}$ & 2015 & $978-84-675-7638-2$ \\
\hline & $\bar{G}$ & Vicens Vives & 2015 & $978-84-682-3045-0$ \\
\hline & $\mathrm{H}$ & Anaya & 2015 & $978-84-678-5219-6$ \\
\hline \multirow{4}{*}{$4^{\circ} \mathrm{ESO}$} & $\bar{I}$ & Santillana & 2016 & $978-84-680-3850-6$ \\
\hline & $\mathrm{J}$ & SM & 2016 & $978-84-675-8697-8$ \\
\hline & K & Vicens Vives & 2016 & $978-84-682-3663-6$ \\
\hline & $\mathrm{L}$ & Anaya & 2016 & $978-84-698-1075-0$ \\
\hline \multirow{4}{*}{$\begin{array}{c}1^{\circ} \\
\text { Bachillera } \\
\text { to }\end{array}$} & $\mathrm{M}$ & Santillana & 2015 & 978-84-680-1327-5 \\
\hline & $\mathrm{N}$ & SM & 2015 & $978-84-675-7652-8$ \\
\hline & $\mathrm{O}$ & Vicens Vives & 2016 & $978-84-682-3053-5$ \\
\hline & $\mathrm{P}$ & Anaya & 2015 & $978-84-678-2702-6$ \\
\hline \multirow{4}{*}{$\begin{array}{c}2^{\circ} \\
\text { Bachillera } \\
\text { to }\end{array}$} & Q & Santillana & 2016 & $978-84-680-3314-3$ \\
\hline & $\overline{\mathrm{R}}$ & $\mathrm{SM}$ & 2016 & 978-84-675-8719-7 \\
\hline & $\mathrm{S}$ & Vicens Vives & 2016 & $978-84-682-3671-1$ \\
\hline & $\mathrm{T}$ & Anaya & 2016 & 978-84-698-1283-9 \\
\hline
\end{tabular}

